\title{
Crossed wiring closure technique for bilateral transverse thoracosternotomy is associated with less sternal dehiscence after bilateral sequential lung transplantation
}

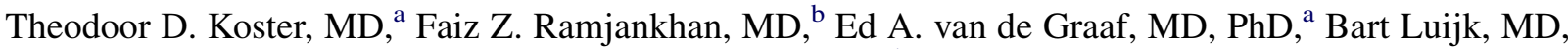 \\ $\mathrm{PhD},{ }^{\mathrm{a}}$ Diana A. van Kessel, MD, ${ }^{\mathrm{c}}$ Ronald C. A. Meijer, MD, ${ }^{\mathrm{b}}$ and Johanna M. Kwakkel-van Erp, MD, PhD ${ }^{\mathrm{a}}$
}

\begin{abstract}
Objective: Bilateral transverse thoracosternotomy (clamshell incision) is a widely used approach in bilateral sequential lung transplantation, but the closure technique is associated with sternal dehiscence. This study compares the incidence of sternal dehiscence between the crossed and uncrossed closure techniques.
\end{abstract}

\begin{abstract}
Methods: In 129 patients who underwent transplantation through a clamshell incision, the sternum was closed using either the crossed or the uncrossed method based on the surgeon's preference. The position of the sternal parts was evaluated on lateral chest radiographs and scored as normal, override, or separation.

Results: We observed sternal override in 38 patients and separations in 18 patients. The sternum was closed using the uncrossed method in 79 patients and the crossed method in 50 patients. There were significantly fewer overrides $(n=6,12.0 \%)$ and separations $(n=6,12.0 \%)$ of the sternal parts using the crossed closure technique compared with the uncrossed technique (32 overrides, $41.0 \%$; and 12 separations, $15.1 \% ; P<.001$ ). Reconstructive surgery was only performed in patients with separation of the sternal parts $(\mathrm{n}=10)$.
\end{abstract}

Conclusions: Using the crossed closure technique for the sternum after bilateral sequential lung transplantation reduces the incidence of sternal dehiscence compared with the uncrossed closure technique and, therefore, reduces the necessity of reconstructive surgery. (J Thorac Cardiovasc Surg 2013;146:901-5)

Bilateral sequential lung transplantation performed through a bilateral transverse thoracosternotomy (the clamshell incision) is a widely used technique that was initially described by Bisson and Bonnette in 1990 and published in this journal in 1992. ${ }^{1,2}$ This clamshell incision includes a transverse division of the sternum at the third or fourth intercostal space and provides excellent exposure of the hilar structures, the mediastinum, and the pleural spaces. ${ }^{3-7}$ Bilateral lung transplantation is performed sequentially. This procedure offers the opportunity for single-lung ventilation during the procedure, eliminating the need for cardiopulmonary bypass in some cases. However, the use of cardiopulmonary bypass is frequently required, and the clamshell incision provides good access for cannulation of the ascending aorta and the right atrium. ${ }^{4,6}$

After transplantation, the sternum is usually closed with 2 or 3 uncrossed loops of stainless steel wire. ${ }^{3-5,8-10}$ Although this closure method is effective in preventing distraction of

\footnotetext{
From the Departments of Respiratory Medicine ${ }^{\mathrm{a}}$ and Cardiothoracic Surgery, ${ }^{\mathrm{b}}$ University Medical Centre Utrecht, Utrecht, The Netherlands; and the Department of Respiratory Medicine, ${ }^{\mathrm{c}}$ St Antonius Hospital, Nieuwegein, The Netherlands. Disclosures: Authors have nothing to disclose with regard to commercial support. Received for publication Sept 22, 2012; revisions received April 4, 2013; accepted for publication April 18, 2013; available ahead of print June 13, 2013.

Address for reprints: Theodoor D. Koster, MD, Department of Respiratory Medicine, University Medical Center Utrecht, Heidelberglaan 100, 3584 CX Utrecht, The Netherlands (E-mail: th.d.koster@gmail.com). $0022-5223 / \$ 36.00$

Copyright (c) 2013 by The American Association for Thoracic Surgery http://dx.doi.org/10.1016/j.jtcvs.2013.04.033
}

the sternal parts, it does not prevent anteroposterior displacement of these parts, which can result in sternal dehiscence. The incidence of sternal dehiscence after bilateral sequential lung transplantation ranges from $8 \%$ to $36 \%{ }^{3-5,8,9}$ This wide range is probably the result of different definitions of sternal dehiscence and closure techniques.

Reconstructive surgery is necessary in some patients with symptoms due to sternal dehiscence, mostly complaints of pain or a clicking sensation. Several wiring techniques with different materials have been used to prevent sternal dehiscence (a sternal fixation device, ${ }^{3}$ reinforced wiring, ${ }^{4}$ Kirschner wires or Steinmann pins, ${ }^{5,9}$ and a peristernal cable system ${ }^{10}$ ). However, these new materials are expensive, and the removal of these osteosynthetic materials may be necessary. ${ }^{4}$

Numerous wiring techniques have been evaluated for median sternotomies, including several parasternal and transsternal closure techniques. ${ }^{11}$ More stable closure is reported with oblique tension on the sutures and some form of lateral support. ${ }^{11}$ However, only few studies regarding the closure technique of the bilateral transverse thoracosternotomy have been performed..$^{3-5,10,12,13}$ In our clinic, several methods are used to close the sternum. In addition to the conventional uncrossed method, we predominantly used the crossed closure methods (the sternally crossed closure) and made a modification of this technique by closing it parasternally (the parasternally crossed method). 

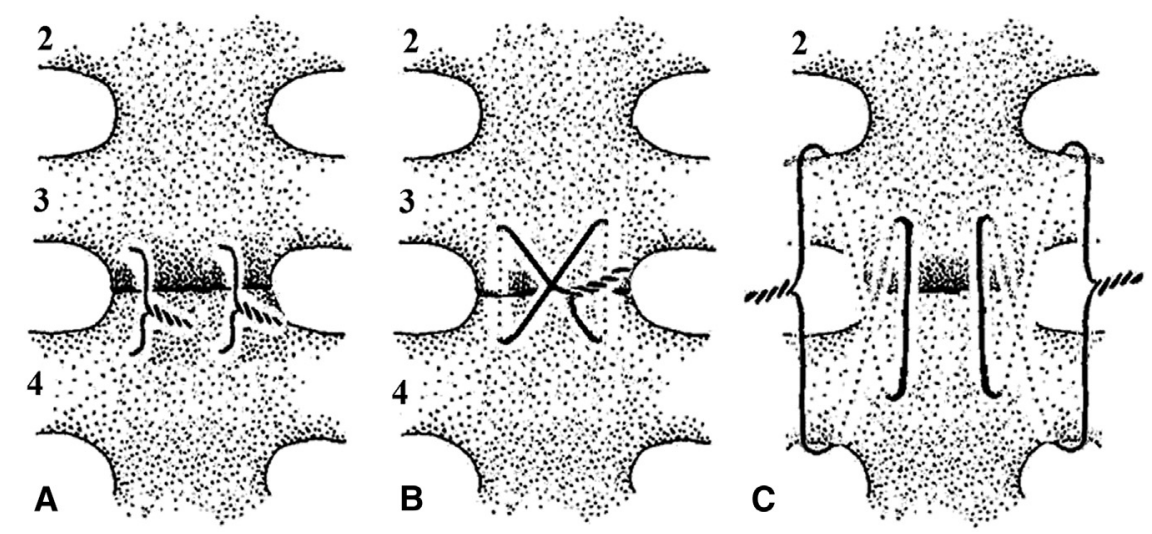

FIGURE 1. Different wiring techniques. Schematic showing different sternal closure wiring techniques for transverse sternotomy at the fourth intercostal space: uncrossed closure technique (A), sternally crossed closure technique (B), and parasternally crossed closure technique (C).

The purpose of this study was to compare the conventional, uncrossed closure technique with the crossed closure technique regarding the incidence of sternal dehiscence and surgical interventions after bilateral lung transplantation. We hypothesized that the crossed closure method diminishes the incidence of sternal dehiscence, thereby reducing the need for corrective surgery.

\section{METHODS \\ Patients}

Patients undergoing lung transplantation via a clamshell approach from January 2001 to February 2011 at the University Medical Center Utrecht (Utrecht, The Netherlands) were included in this retrospective cohort study. All patients received standard immunosuppressive therapy consisting of basiliximab, tacrolimus, mycophenolate mofetil, and prednisone. Medical records were checked for demographic information and preoperative, perioperative, and postoperative data. The study was approved by the medical ethical committee, and informed consent was obtained from each patient.

\section{Closure Technique}

The closure techniques used for the bilateral transverse thoracosternotomy were either the uncrossed wiring technique with 2 or 3 steel wires (uncrossed group) or the crossed wiring technique (crossed group). When implementing the crossed closure technique, we used either the parasternal or the sternal crossing technique of the steel wires (Figure 1). The sternally crossed closure technique involves closure with 1 or 2 figureof-eight steel wires across the sternum. A parasternally crossed closure technique is closure with 2 figure-of-eight steel wires across the sternum and around the nearest ribs cranial and caudal to the sternal cleavage site. The chosen closure technique was based on the surgeon's preference.

\section{Imaging}

Chest radiographs were obtained and analyzed at 1, 6, and 12 months after transplantation. The position of the sternum was evaluated using the lateral chest radiographs, and scored as normal, override, or separation. Sternal override is a partial displacement but maintained overlap of the 2 sternal parts, whereas sternal separation is the complete disruption of the 2 sternal parts without remaining overlap of the 2 sternal parts. Overrides or separations of the sternal parts were both defined as sternal dehiscence (Figure 2). The posterior anterior chest radiograph was used to score the closure technique as either crossed or uncrossed (Figure 1). Patients who died before a lateral chest radiograph was performed were excluded from the study.

\section{Statistical Analysis}

Data were analyzed using PASW Statistics software version 17.0.2 (SPSS Inc, Chicago, Ill) and GraphPad Prism software version 5.03 (GraphPad Software, San Diego, Calif). Comparisons between categorical variables were performed using the Pearson $\chi^{2}$ or the Fisher exact test, when appropriate. Continuous data were analyzed with a 1-way analysis of variance or an unpaired $t$-test for parametric variables. The MannWhitney or the Kruskal-Wallis test was used for nonparametric variables. Multivariate analyses were performed by using a stepwise logistic regression, in which all variables with $P<.10$ in the univariate analyses were included. $P<.05$ was considered significant.

\section{RESULTS \\ Study Population}

From January 2001 to February 2011, we performed 184 lung transplantations. In 35 patients, single-lung transplantation was performed through a posterolateral thoracotomy. In 149 patients, lung transplantation was performed via a clamshell incision. Twenty patients died postoperatively before a lateral chest radiograph was obtained and were excluded from analysis. The causes of death were as follows: excessive bleeding and hypovolemic shock $(\mathrm{n}=10)$; sepsis, acute respiratory distress syndrome, or multiorgan failure

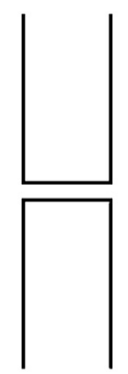

Normal

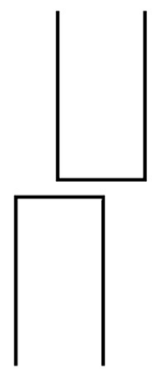

Override

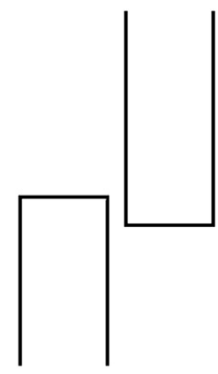

Separation
FIGURE 2. Sternum position. The position of the sternum on the lateral chest radiograph was scored as normal, override, or separation. 
TABLE 1. Characteristics of the study group

\begin{tabular}{|c|c|c|c|c|}
\hline Characteristics & $\begin{array}{c}\text { Uncrossed } \\
(\mathbf{n}=79)\end{array}$ & $\begin{array}{l}\text { Crossed } \\
(\mathbf{n}=\mathbf{5 0})\end{array}$ & $\begin{array}{c}\text { Total } \\
(\mathbf{n}=\mathbf{1 2 9})\end{array}$ & $P$ value \\
\hline Male sex & $37(47)$ & $29(58)$ & $66(51)$ & .219 \\
\hline Age, y & $42 \pm 13$ & $44 \pm 14$ & $43 \pm 13$ & .683 \\
\hline Indications & & & & .864 \\
\hline $\mathrm{CF}$ & $29(37)$ & $15(30)$ & $44(34)$ & \\
\hline COPD & $32(40)$ & $22(44)$ & $54(42)$ & \\
\hline ILD & $18(23)$ & $13(26)$ & $31(24)$ & \\
\hline $\begin{array}{l}\text { Mean surgical procedure } \\
\text { time, } \min \end{array}$ & $312 \pm 84$ & $332 \pm 66$ & $320 \pm 77$ & .671 \\
\hline Mean ICU stay, d & $8 \pm 8$ & $7 \pm 9$ & $7 \pm 8$ & .947 \\
\hline
\end{tabular}

$(\mathrm{n}=5)$; fatal cerebral damage $(\mathrm{n}=3)$; or massive lung bleeding caused by a bronchovascular fistula $(\mathrm{n}=2)$. Death was not related to sternal complications. There were no documented sternal infections in the study group.

From the 129 patients who underwent a bilateral transverse thoracosternotomy, lung transplantation was performed bilaterally in 126, and in 3 patients, bilateral transplantation was converted to single-lung transplantation because of the presence of massive pleural adhesions. The baseline patient characteristics are shown in Table 1. Cardiopulmonary bypass or Veno-Arterial Extracorporeal Life Support was required in $61 \%$ of the patients. In 79 patients, the uncrossed closure technique was used to close the bilateral transverse thoracosternotomy (71 patients received 2 steel wires, and 8 patients received 3 steel wires; Surgical Steel Ethicon 653B, Ethicon, Inc, Somerville, NJ), whereas the crossed closure technique was used in 50 patients. Reoperations for bleeding in the immediate postoperative period were required in 18 patients, 12 of which were performed by reopening the clamshell incision.

The 2 study groups were similar with respect to sex, age, underlying disease, use of cardiopulmonary bypass, surgical procedure time, need for reoperation for bleeding, and length of stay in the intensive care unit.

\section{Sternal Dehiscence}

In $56(43 \%)$ of the 129 patients, sternal dehiscence was found. An override was diagnosed in 38 patients $(30 \%)$, and a separation of the sternum was diagnosed in 18 patients $(14 \%)$. An override was observed in $32(41 \%)$ of the 79 patients in the uncrossed group, and a separation of the sternum was observed in 12 patients (15\%). In comparison, 6 $(12 \%)$ of the 50 patients had an override and $6(12 \%)$ had a separation of the sternum in the crossed group $(P<.001)$ (Figure 3). To determine whether parasternally crossed closures are superior to sternally crossed closures, the crossed closure group was subdivided into 2 different groups: sternally crossed (33 patients) and parasternally

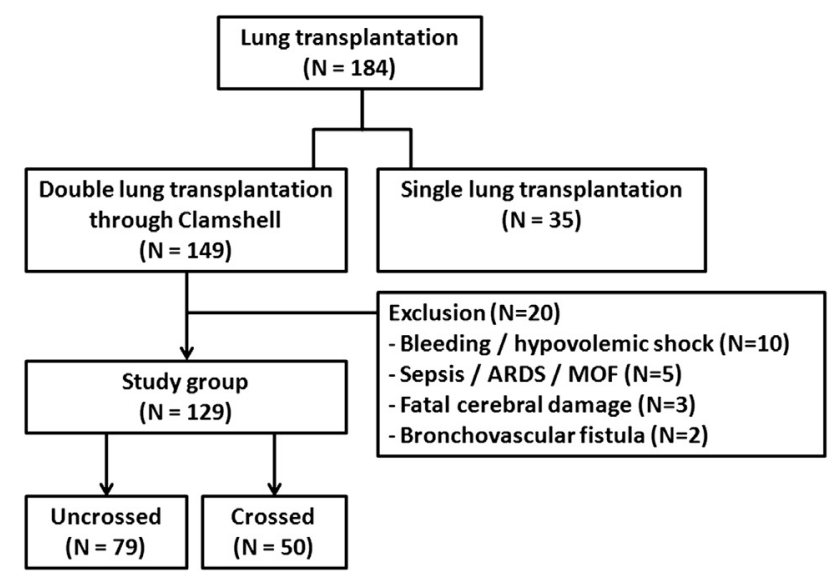

FIGURE 3. Flow chart of study group.

crossed (17 patients). There was 1 override and no separations in the parasternally crossed group, compared with 5 overrides and 6 separations in the sternally crossed group $(P=.08)$ (Figure 4).

The initial diagnosis, age, sex, use of cardiopulmonary bypass, surgical procedure time, and requirement for reoperation through the clamshell incision (to control postoperative bleeding) did not prove to be significant risk factors for developing sternal dehiscence.

\section{Reconstructive Surgery}

Reconstructive surgery was performed in $10(8 \%)$ of the 129 patients. All of the corrections were performed in patients with sternal separation (10 of 18) accompanied by pain, and no corrections were performed in patients with sternal overrides $(P<.0001)$. Patients with sternal override did not have a clicking sensation. Some patients did experience pain, but with adequate pain medication, these complaints faded away. Of the 79 patients in the uncrossed group, $7(9 \%)$, and $3(6 \%)$ of the 50 patients in the crossed group received surgery. No corrective surgery was required in the parasternally crossed group, because there were no separations in this group.

Although sternal separation in 18 patients was visible on a lateral chest radiograph at 12 to 84 (median, 24) days after transplantation, the time to corrective surgery ranged from 25 to 1017 (median, 134) days.

\section{DISCUSSION}

Sternal dehiscence is an important complication after bilateral transverse thoracosternotomy. Our data indicate that the crossed closure technique reduces the incidence of sternal dehiscence after sternotomy. Herein, we describe a new wiring technique for sternal closure after bilateral transverse thoracosternotomy (the parasternally crossed closure technique) that decreases the incidence of sternal dehiscence and the need for reconstructive surgery. 


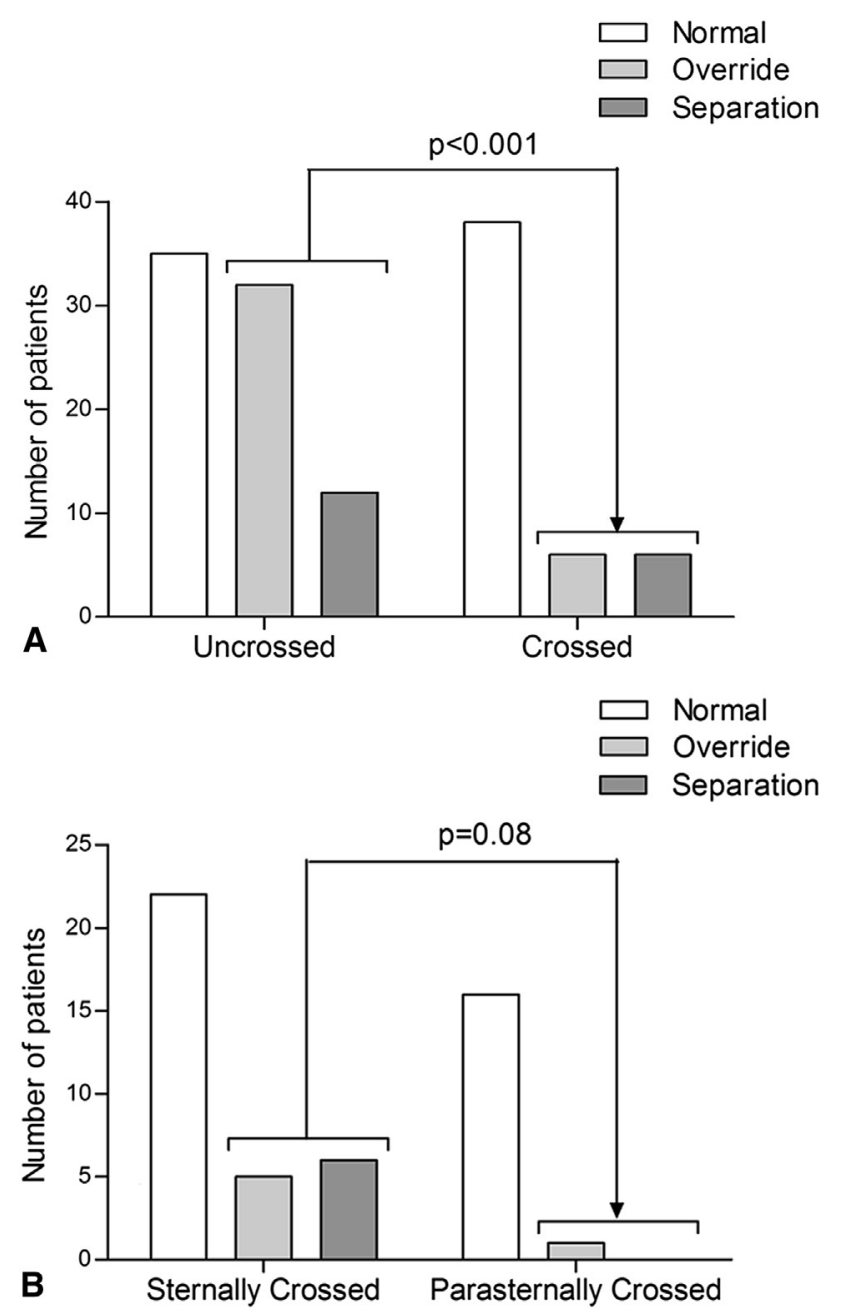

FIGURE 4. A, Uncrossed versus crossed closure technique. In the 2 different closure groups, the numbers of patients with normal position of the sternum, override, or separation are presented. The crossed closure method reduces the incidence of override and separation compared with the uncrossed closure method $(P<.001)$. B, Sternally crossed versus parasternally crossed. The crossed closure method is subdivided into a sternally and parasternally crossed closure technique. The numbers of patients with override and separation in the 2 groups are presented. A trend toward less override and separation is observed in the parasternally compared with the sternally crossed closure technique $(P=.08)$.

In agreement with our results, other authors acknowledge the problem of sternal dehiscence with the uncrossed method. ${ }^{3-5,8,9,12}$ Brown and colleagues ${ }^{3}$ described a sternal disruption rate of $36 \%$, and Macchiarini and colleagues ${ }^{8}$ found sternal override in $32 \%$ of cases. These authors did not, however, describe their definitions and methods of measurement. Oto, ${ }^{4}$ Meyers, ${ }^{5}$ and Venuta ${ }^{9}$ and colleagues reported the incidence of patients requiring surgical intervention as $23 \%, 8 \%$, and $8 \%$, respectively. These studies emphasize the necessity of using uniform terminology for sternal dehiscence. We suggest that sternal dehiscence be defined as the separation of the sternal parts, and the diagnosis can be made on a lateral chest radiograph. In fact, only sternal separation, and not sternal override, requires surgical correction.

In our study, we demonstrated that the crossed closure technique significantly reduces sternal dehiscence compared with the uncrossed technique. The success of the crossed closure technique may be because of the attainment of a more stable closure, with oblique tension on the sutures and an equal distribution of traction. ${ }^{12}$ The parasternally crossed closure may be superior to the sternally crossed closure because the former technique applies partial tension to the thoracic cage rather than to the sternum alone.

Other options to prevent the problem of sternal dehiscence, in addition to the different closure techniques of the bilateral transverse thoracosternotomy, may lay in approaching the sternum. Lonchyna ${ }^{13}$ suggested an inverted V-shape transsection of the sternum to improve stability and a crossed closure technique with 2 steel wires; unfortunately, he did not describe numbers of overriding or dislocation. Some authors suggest the bilateral anterior thoracotomy without sternal cleavage as an alternative to the full clamshell incision. ${ }^{5,8,9}$ Because this approach does not require sternal division, there is no risk of sternal dehiscence. If cardiopulmonary bypass is needed, cannulation of the aorta can be performed using the Seldinger technique. Right atrium cannulation is also possible through a right anterior thoracotomy or even through cannulation of the femoral vessels. ${ }^{9,14}$ Avoiding sternal cleavage also prevents other complications due to the clamshell incision, such as costochondrial infections and wound dehiscence. Unfortunately, we did not investigate these aspects in our study group. Sternal pain and discomfort and possibly paradoxal breathing are also associated with a clamshell approach, but in our experience could be managed adequately with pain medication.

Because our transplantation center is a large referral center for patients with a diagnosis of cystic fibrosis, we see many patients with pleural adhesions due to this underlying disease or as a consequence of pretransplantation procedures performed for pneumothoraxes. The clamshell incision is still the most frequently used technique when a difficult dissection or the need for cardiopulmonary bypass is anticipated, also in studies using the clamshellsparing method. ${ }^{4,5,9,10,13}$ For these reasons, the clamshell incision is the surgical preference in our clinic because of a low threshold for installation of cardiopulmonary bypass to facilitate transplantation. More recently, we use VenoArterial Extra Corporeal Life Support when cardiopulmonary bypass is needed. ${ }^{15}$

Because this study was a retrospective cohort study, there was no randomization of closure methods. Although the chosen closure technique was based on the surgeon's preference and not linked to specific patient characteristics or risk factors, patient selection bias is unlikely 
because baseline characteristics were not different between groups.

Twelve different surgeons performed closure of the sternum during this study period; most of them used several closure techniques. Unfortunately, the data could not be corrected for possible confounding factors, such as learning curves or individual skill differences between surgeons. However, because the incidence of sternal dehiscence could not be linked to specific surgeons and all surgeons used several closure techniques, bias seems unlikely.

In conclusion, closure of the bilateral transverse thoracosternotomy using the crossed wiring technique, especially the parasternally crossed method, reduces the incidence of sternal dehiscence. This method of closure is adopted in our clinic, and we recommend this closure method over the uncrossed method to prevent sternal dehiscence, which is defined as separation of the sternal parts.

\section{References}

1. Bisson A, Bonnette P. A new technic of bipulmonary transplantation: double unilateral lung transplantation. Presse Med. 1990;19:762.

2. Bisson A, Bonnette P. A new technique for double lung transplantation: "bilateral single lung" transplantation. J Thorac Cardiovasc Surg. 1992;103: 40-6.

3. Brown R, Esmore D, Lawson C. Improved sternal fixation in the transsternal bilateral thoracotomy incision. J Thorac Cardiovasc Surg. 1996;112: 137-41.
4. Oto T, Venkatachalam R, Morsi Y, Marasco S, Pick A, Rabinov M, et al. A reinforced sternal wiring technique for transverse thoracosternotomy closure in bilateral lung transplantation: from biomechanical test to clinical application. J Thorac Cardiovasc Surg. 2007;134:218-24.

5. Meyers B, Sundaresan R, Guthrie T, Cooper J, Patterson G. Bilateral sequential lung transplantation without sternal division eliminates posttransplantational sternal complication. J Thorac Cardiovasc Surg. 1999;117:358-64.

6. Parekh K, Patterson G. Technical considerations in adult lung transplantation. Semin Thorac Cardiovasc Surg. 2004;16:322-32.

7. Bains M, Ginsberg R, Jones W, McCormack P, Rusch V, Burt M, et al. The clamshell incision: an improved approach to bilateral pulmonary and mediastinal tumor. Ann Thorac Surg. 1994;58:30-2.

8. Macchiarini P, Le Roy Ladurie F, Cerrina J, Fadel E, Chapelier A, Dartevelle P. Clamshell or sternotomy for double lung or heart-lung transplantation? Eur J Cardiothorac Surg. 1999;15:333-9.

9. Venuta F, Rendina E, De Giacomo T, Ciccone A, Moretti M, Mercadante E, et al. Bilateral sequential lung transplantation without sternal division. Eur J Cardiothorac Surg. 2003;23:894-7.

10. McGiffin D, Alonso J, Zorn G, Kirklin J, Randall Young K, Wille K, et al. Sternal approximation for bilateral anterolateral transsternal thoracotomy for lung transplantation. Ann Thorac Surg. 2005;79:19-20.

11. Losanoff JE, Jones JW, Richman BW. Primary closure of median sternotomy: techniques and principles. Cardiovasc Surg. 2002;10:102-10.

12. Wright C. Transverse sternothoracotomy. Chest Surg Clin NAm. 1996;6:149-56

13. Lonchyna V. Sternal "notching" improves clamshell incision. Operat Tech Thorac Cardiovasc Surg. 1999; 4:176-8.

14. Varela A, Montero C, Castedo E, Roda J, Gámez P, Madrigal L, et al. Transcutaneous extracorporeal cannulation for bilateral lung transplantation without splitting the sternum. J Thorac Cardiovasc Surg. 2000;119:402-3.

15. Ius F, Kuehn C, Tudorache I, Sommer W, Avsar M, Boethig D, et al. Lung transplantation on cardiopulmonary support: venoarterial membrane oxygenation outperformed cardiopulmonary bypass. J Thorac Cardiovasc Surg. 2012;144: 1510-6. 